\title{
Domain Ontology, an Instrument of Semantic Web Knowledge Management in E-Learning
}

\author{
http://dx.doi.org/10.3991/ijac.v5i4.2288 \\ A.J.Gladun ${ }^{1,2}$, J.V. Rogushina ${ }^{2}$ and J. Schreurs ${ }^{3}$ \\ ${ }^{1}$ Ministry of Education, Kyiv, Ukraine \\ ${ }^{2}$ National Academy of Sciences, Kyiv, Ukraine \\ ${ }^{3}$ Hasselt University, Hasselt, Belgium
}

\begin{abstract}
Domain ontology is proposed as an instrument for controlling of e-learning course results. This approach instead of traditional testing more objectively reflects the structure of students' knowledge. Use of ontologies for knowledge representation provides interoperability of the used testing systems. The domain knowledge has been found and reuse in the semantic web world. Domain ontology developing is based on the principles of ontological analysis. The method of it's use for evaluation of the learners' knowledge results is based on matching of the learners' ontology with the reference ontology proposed by the trainer/tutor. A multi-agent system for e-learning (M(e)L ) has been defined and a prototype system has been developed. In a case, the domain ontology and the semantic web representation has been applied in two e-learning university courses.
\end{abstract}

Index Terms - ontology, knowledge management, intelligent agent, e-learning, Semantic Web.

\section{INTRODUCTION: DOMAIN ONTOLOGY AS AN INSTRUMENT OF E-LEARNING}

\section{A. E-learning is improving the learning quality}

Modern computer technologies significantly changed the training practice. Use of multimedia, simulation, computer-mediated communication and communities, and internet-based support for individual and distance learning all have the potential for revolutionary improvements in training $[1,2]$.

Online learning (e-learning) now is very popular. It offers new important possibilities in training: a learner can become immediate feedback from solutions to problems, learning paths can be highly individualized and the learned information is actual (based on current facts, technologies and scientific efforts), etc.

Now oline learning is a rapidly growing business: the number of organizations working on online learning and the number of courses available on the Internet is growing rapidly $[3,4]$.

\section{B. Transformation of trainer-learner interaction in the distance/online learning process based on a tutor system. The problem of examination}

E-learning is an alternative concept to traditional training. Control of the learners' learning activities by an online tutoring system seems to be relatively weaker compared with the traditional control where a tutor is in charge of the learning content and the organization of the learning process via face to face sessions. In a tutoring system the problem of knowledge management is much more complex because a trainer can't interact with every learner on all phases of learning and cannot take into account the cognitive style, level of training and other specific features of the learner.

So, e-tutoring system has to include some automated tools to control learners' skills - not only formally (for example by testing) but also on semantic level.

An online e-tutoring system should emphasize engaging learners in the learning process and has to be adaptive to each individual learner.

The goal of the early software tutoring systems was to build user interfaces that provide the efficient access to knowledge for the individual learners. Recent and emerging work focuses on the learner control over the learning process such as learner exploring, designing, constructing, and using adaptive systems as tools $[5,6]$.

With the application of more computer techniques in education and the involvement of more adults in software tutoring systems, the learner control strategy has become more appreciated than tutor control or program control.

Learners' skills and knowledge have to be tested. One of the main problems in testing is the required interoperability of tests in different testing systems. (To be able to exchange tests between various systems it is necessary to create some universal format of tests preservation and their processing instructions.).

Another problem is the fact that the result of a test is not always reflecting the real students' knowledge of the domain. It is well known that in traditional tests mistakes can be involved as a consequence of an ambiguous formulation of the questions or of misprints and even worse that correct answers can be obtained intuitively or by accident.

Learning control needs the instrument to compare learner's knowledge resulting from learning in the learning process with the course domain knowledge as being expected as a result of learning. It requires the powerful and interoperable tools of knowledge representation and analysis.

In an online learning process, the automation of the knowledge evaluation and its objectivity is a main issue.

The solution for the problem will be on point of test semantic. Many authors [7,8] utilize the ontology's semantic data to improve the reading and analyses of information in unstructured documents. Using domain ontology as an instrument to evaluate the learners' knowledge is proposed. 


\section{Using domain ontology to analyze the learning result of the individual learner}

Human readable data resources (like control of learner's work, reports etc.) are not easy to control by automated machine processing. A structured information representation of ontology (machine-processed representations containing the semantic information of a domain) is required and will be very useful to represent the knowledge on a formal way.

Ontology is based on descriptive logics. It can be interpreted and analyzed by various reasoning systems. Now a lot of standards, languages and software tools for ontological analyses are always proposed by Semantic Web project and widely used in different intelligent Web applications.

The domain ontology plays a central role as a resource structuring the learning content [9]. One of the key challenges of the course construction process is to identify the abstract domain of information within which this course will exist. The trainer has to describe the content of the course in content modules.

The main idea of our approach is that the domain ontology is not only an instrument of learning (searching knowledge) but also a tool of examination of the learners' knowledge.

Learners will build their domain ontology and then compare it - in automated mode - with the reference one. As a result the system will show the parts of domain knowledge which are misunderstood and by the way it will support the trainer while improving the online course.

Experiments are demonstrating that this approach of analysing the semantics of the domain is much more efficient then usual tests.

\section{SEMANTIC WEB AND EFFICIEN ACCESS TO THE WEB KNOWLEDGE}

The Semantic Web is the new generation of the World Wide Web, based on the semantic network knowledge representation formalism, which enables packaging information in the form of object-attribute-value statements, the so called triplets. The vision of the Semantic Web proposes more intelligent services by facilitating machine understanding of the content.

\section{A. Main features of the Semantic Web project}

Semantic Web is used as an appropriate infrastructure for intelligent agents to run around the Web and performing complex actions for their users [12].

Furthermore, Semantic Web is about explicitly explaining the knowledge embedded in many web-based applications, integrating information in an intelligent way, providing semantic-based access to the Internet, and extracting information from texts [13].

Semantic Web provides automated information access thanks to machine processing of data based on the semantics and on heuristics using metadata $[14,15]$.

An important advantage of the Semantic Web is that people that use Web resources can collaboratively create ontologies and build common vocabulary without centralized control. Ontologies applied to the Web are creating the distributed knowledge base of Semantic Web [19].

\section{B. Ontological representation of knowledge}

Ontology is an important building block of knowledge in the Semantic Web. Ontological approach to knowledge representation provides a shared and common understanding of domain that can be communicated across people and applications. It comprises a set of knowledge terms, including the vocabulary, the semantic interconnections, and some simple rules of inference and logic for some particular topic [16].

Ontologies propose the necessary armature around which knowledge bases should be built [17], and set grounds for developing reusable Web-contents, webservices and applications [18].

Ontologies facilitate knowledge sharing and reuse, i.e. a common understanding of various contents that reaches across people and applications.

Knowledge in ontology is mainly formalized using five kinds of components: classes, relations, functions, axioms and instances [3].

\section{Semantic Web and machine-readable and global access to knowledge and learning content}

The explicit representation of the semantics of data, accompanied with domain theories (ontology), will enable a Web that provides a qualitatively new level of service, such as: intelligent search engines, information brokers, and information filters [20].

If a learning contents frame of learning resources can be introduced into an e-learning system, including ontologybased properties and hierarchical semantic associations, then this e-learning system will have the more efficient capabilities of providing adaptable and intelligent learning to learners.

Availability of formal model of ontology is playing a crucial role in enabling the representation, processing, sharing and reusing knowledge between applications in a web-based e-learning system, because it specifies the conceptualization of a specific domain in terms of concepts, attributes, and relationships.

The ontological knowledge is added to the learning resources as a resource for contextual learning, and it can be searched by means of queries.

\section{Semantic Web technologies as a platform for e- learning}

Semantic Web can be used as a very suitable platform for implementing an e-learning system, because it provides all means for (e-learning): ontology development, ontology-based annotation of learning materials, their composition in learning courses and proactive delivery of the learning materials through elearning portals.

A personal ontology can in future be used for group formation: gather learners with similar thesauri and ontology.

\section{MODEL OF ONTOLOGY, ONTOLOGICAL ANALYSIS AND DEVELOPMENT OF ONTOLOGIES}

\section{A. Definitions and formal model of ontology}

Domain ontology is an instrument for knowledge representation, sharing, reuse and interoperability, having an increasingly important role in the process of designing 
personalized intelligent e-learning architectures and systems.

Definitions of ontology differ from researcher to researcher.

Some of the definitions, used in the computer science field, can be summarized as follows: ontology is:

1) "a representation of a conceptual system that is characterized by specific logical properties";

2) "a synonym of conceptualization";

3) "a conceptual specification that describes knowledge about domain in a manner that is independent of epistemic states and state of affairs";

4) "the study of what exists in a given domain or universe of discourse";

5) "a special kind of knowledge bases".

Ontology includes a catalogue of terms used in a course domain, managing the rules governing how those terms can be combined to make valid statements about situations in that domain, and the sanctioned inferences that can be made when such statements are used in that domain.

In the context of ontology, a relation is a definite descriptor referring to an association in the real world; a term is a definite descriptor that refers to an object or situation-like thing in the real world.

The formal model of ontology $\mathrm{O}$ is an ordered triple of finite sets:

$\mathrm{O}=<\mathrm{T}, \mathrm{R}, \mathrm{F}>[23]$,

where

- $\quad \mathrm{T}$ - the set of the domain concepts and terms which is described by ontology $\mathrm{O}$;

- $\quad \mathrm{R}$ - finite set of the relations between terms of domain;

- $\mathrm{F}$ - the domain interpretation functions on the terms and the relations of ontology $\mathrm{O}$.

In process of ontology building learners use relations from the fixed set that contains the most widely used relations from $\mathrm{R}$ :

- Hierarchical relations:

"is a subclass of",

"is a part of",

"has attributes",

"has elements";

- Relations of similarity

"is a synonym",

"is near to".

\section{B. Basic Principles of ontological Analysis}

Ontological analysis is accomplished by examining the vocabulary that is used to discuss the characteristic objects and processes of the domain, developing rigorous definitions of the basic terms in that vocabulary, and characterizing the logical connections among those terms.

The product of this analysis, an ontology, is a some domain vocabulary completed with a set of precise definitions, or axioms, that constrain the meanings of the terms sufficiently to enable consistent interpretation of the data that use that vocabulary.

\section{Main steps of ontology construction}

Building of ontology is based on the IDEF5 methodology [22].

Following the IDEF5 method, learners must perform three tasks:

1) Build the set of the domain terms;

2) Capture the constraints that govern how those terms can be used to make descriptive statements about the domain;

3) Build a model that, when provided with a specific descriptive statement, can generate the "appropriate" additional descriptive statements [11].

The learners (as well as the tutor) have to execute four main steps to design the ontology of domain:

1) Define the main classes and terms of domain and describe their meaning:

- Define the set of class names T;

- Define the set of relation names R;

- For every class name define the set of attribute names At;

- For every attribute name $a \in A_{t}, t \in T$ define it type - INT, STRING, NUMBER ets. or other class of ontology;

2) Construct the taxonomy of domain terms:

- Define all pairs of classes

- $<\mathrm{t}_{1}, \mathrm{t}_{2}>, \mathrm{t}_{1} \in \mathrm{T}, \mathrm{t}_{2} \in \mathrm{T}, \mathrm{r}\left(\mathrm{t}_{1}, \mathrm{t}_{2}\right) \rightarrow$;

$\mathrm{t}_{1}$ "IS_A_Subclass_Of" $\mathrm{t}_{2}, \mathrm{r} \in \mathrm{R}$

3) Define synonymy and other relations between these terms:

- Define all pairs of classes

- $<\mathrm{t}_{1}, \mathrm{t}_{2}>, \mathrm{t}_{1} \in \mathrm{T}, \mathrm{t}_{2} \in \mathrm{T}, \mathrm{r}\left(\mathrm{t}_{1}, \mathrm{t}_{2}\right) \rightarrow$;

$\mathrm{t}_{1}$ "IS_Synonyme_Of" $\mathrm{t}_{2}, \mathrm{r} \in \mathrm{R}$

- Define all pairs of classes

- $<\mathrm{t}_{1}, \mathrm{t}_{2}>, \mathrm{t}_{1} \in \mathrm{T}, \mathrm{t}_{2} \in \mathrm{T}, \mathrm{r}\left(\mathrm{t}_{1}, \mathrm{t}_{2}\right) \rightarrow$;

$\mathrm{t}_{1}$ "Related_With" $\mathrm{t}_{2}, \mathrm{r} \in \mathrm{R}$

4) Describe the instances of constructed classes:

- Define names of instances a;

- Define meanings of all attributes of instance class $\forall a \in t, t \in T$.

\section{Development of ontology}

In view of knowledge interoperability technologies developed in Semantic Web project [24] are applied. The ontologies are stored in a semantic markup language OWL [17] that is developed as a vocabulary extension of RDF [16] for applications that process the content of information.

The OWL Web Ontology Language is being designed by the W3C Web Ontology Working Group as a revision of the DAML+OIL web ontology language.

The OWL syntax has a frame-like style, where a collection of information about a class or property is given in one large syntactic construct, instead of being divided into a number of atomic chunks for ease of readability. An OWL ontology is a sequence of axioms and facts, plus 
inclusion references to other ontologies, which are considered to be included in the ontology. OWL ontologies are web documents, and can be referenced by means of a URI. Ontologies also have a non-logical component that can be used to record authorship, and other non-logical information associated with a ontology.

$<$ ontology $>\quad:=$ Ontology $([<$ authorship-etc $>]$ $\{<$ directive $>\}$ )

$$
\begin{aligned}
& <\text { authorship-etc }>::=\ldots \\
& <\text { directive }>::=<\text { imports }> \\
& <\text { directive }>::=<\text { axiom }> \\
& <\text { directive }>::=<\text { fact }> \\
& <\text { imports }>::=\text { imports }(<U R I>)
\end{aligned}
$$

Ontologies incorporate information about classes, properties, and individuals, each of which can have an ID which is URI reference. There are two built-in classes in OWL: owl:Thing is the class of all individuals, and owl:Nothing is the empty class. There are two kinds of facts in OWL. The first kind of fact states information about a particular individual, in the form of classes that the individual belongs to plus properties and values of that individual. An individual can be given an individualID that will denote that individual, and can be used to refer to that individual.

OWL is supported by many ontology visualizes and editors, like Protégé 2.0 [21]. Protégé is an integrated software tool used by system developers and domain experts to develop knowledge-based systems. Ontology in Protégé is a model of a particular field of knowledge - the concepts and their attributes, as well as the relationships between the concepts. It is represented as a set of classes with their associated slots.

\section{ONTOLOGY OF DOMAIN OF A COURSE. COMPARING ONTOLOGIES OF LEARNERS WITH A REFERENCE ONTOLOGY OF THE TRAINER/ TUTOR}

\section{A. Ontology of course domain}

The trainer builds the reference course ontology on base of textual documents and his own knowledge in this domain that he has to reflect at materials of distant course. This ontology is proposed for students at the beginning of learning process.

The level of detail is determined by the trainer and course or domain features. Ontology defines the terms used to describe and represent an area of knowledge.

At the 1st step the trainer defines [22]:

- the set of main domain concepts T;

- the properties of each concepts (some properties can be the concepts);

- the 3 sets of relations: hierarchical Rh, equivalence $\mathrm{Re}$ and specific for domain $\mathrm{Rd}$.

At the 2nd step the trainer joins all concepts from $\mathrm{T}$ by relations from $\mathrm{Rh}, \mathrm{Re}$ and $\mathrm{Rd}$ and forms the connected graph (Figure 1).

Then learners receive the sets $\mathrm{T}, \mathrm{Rh}, \mathrm{Re}$ and $\mathrm{Rd}$. Their task is to define the relation between the terms as a trainer does on step 2. This task is not so complex as a development of domain ontology in general, but fixed sets of concepts and relations allow an automated match of learners' ontologies with reference one.

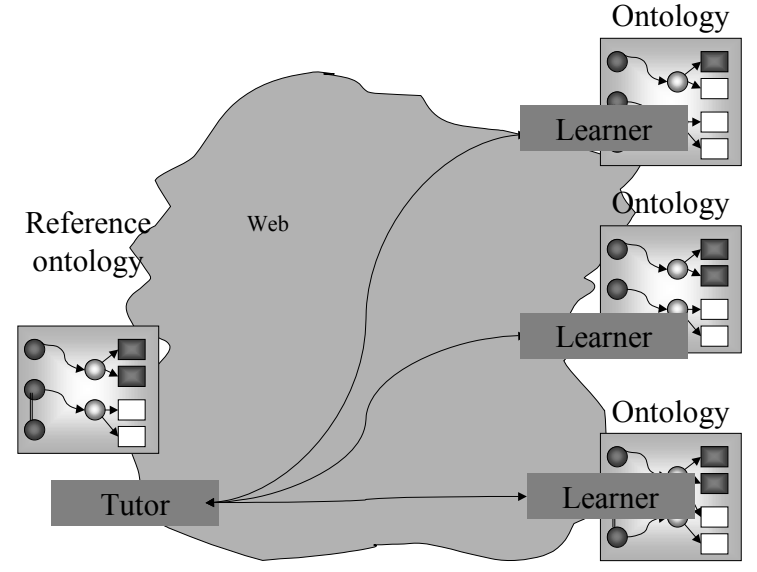

Figure 1. Building of ontology as a result of learning

The purpose in this paper is to design and develop ontology in a learning area that could be used in the provision of an e-learning course. It will be shown in an example course later in the paper.

\section{B. Comparing learners ontology with the reference ontology of the trainer/ tutor}

The trainer compares the domain ontology built by the learner with the reference ontology constructed by the trainer.

Where two similar but different knowledge structures are going to be compared some types of differences can be appear:

- Structural;

- Label (synonyms and homonyms);

- Types of data;

- Levels of metasructure;

- Data types

The original algorithm for automatically comparing of ontologies can be used. It provides the control of correspondence of hierarchical levels in terms of taxonomy (if class $\mathrm{A}$ is a subclass of $\mathrm{B}$ in reference taxonomy and $\mathrm{B}$ is a subclass of $\mathrm{A}$ in learners taxonomy there is a mistake - Fig.5) and it controls affiliation of instances with classes (if instance a belongs to class A in reference taxonomy and learner describes instance a belongs to class B, there is a mistake - Fig.6).

This algorithm is based on some specific conditions and that's why it cannot be used for matching of all arbitrary ontologies:

- A learner has to use ontological terms for classes and subclasses only from the fixed set corresponding to terms of reference ontology, other terms are considered as mistakes;

- A learner has to use relations between classes only from the fixed set corresponding to relations of reference ontology, other relations are considered as mistakes too;

\section{Algorithm of ontology comparison}

We compare the learners' ontology Os with reference ontology Oe made by tutor

1) Define the sets of ontology terms $\mathrm{Ts}$ and $\mathrm{Te}$. 
2) Classify terms from $T s$ on three disjoint categories: Tn, Tu and Tw.

$T_{s}=T_{n} \cup T_{u} \cup T_{w}$ where

- Correctly defined terms $T_{n} \subseteq T_{e}$,

- Not accurately defined terms $T_{u} \not \subset T_{e}$ but $\forall t_{i} \in T_{n} \exists t_{j_{1}} \in T_{e}, \ldots, t_{j_{m}} \in T_{e}, t_{j_{k}} \in T_{e}, m=\overline{1, k}$, and

- Incorrectly defined terms $T_{u} \not \subset T_{e}$ and $\forall t_{i} \notin T_{n} \neg \exists t_{j} \in T_{e}$.

3) Define the sets of ontology relations Rs and Re .

4) Classify relations from Rs on three disjoint categories: $\mathrm{Rn}, \mathrm{Ru}$ and $\mathrm{Rw}$. $R_{s}=R_{n} \cup R_{u} \cup R_{w}$ where

- Correctly defined terms $R_{n} \subseteq R_{e}$,

- Not accurately defined terms $R_{u} \not \subset R_{e}$ but $\forall r_{i} \in R_{n} \exists r_{j_{1}} \in R_{e}, \ldots, r_{j_{m}} \in R_{e}, r_{j_{k}} \in R_{e}, m=\overline{1, k}$ , and

- Incorrectly defined terms $R_{u} \not \subset R_{e}$ and $\forall r_{i} \notin R_{n} \neg \exists r_{j} \in R_{e}$.

Analyze the use of ontology terms and relations. We don't consider the use of terms from $\mathrm{Tw}$ and relations from Rw.

It's very important to take into account the type of relations - hierarchical or improper: Mistake of use "is a part" relation instead of "is a subclass" is much less principle then use "is a synonym" relation instead of "is a subclass" one.

\section{Types of mistakes identified in a learners' ontology}

We distinguish the mistakes of different gravity.

If a learner is using an improper relation from a group of hierarchical relations (for example, $\mathrm{A}$ is a part of $\mathrm{B}$ instead of $A$ is a subclass of $B$ ) it is not so important as if he uses hierarchical relation instead of synonymy relation (for example, A is a part of B instead of A is a synonym of B).

More important mistakes for domain understanding are the error direction in direction of class hierarchy - (for example, $\mathrm{A}$ is a part of $\mathrm{B}$ instead of $\mathrm{B}$ is a part of $\mathrm{A}-$ Fig.2) and instance classification error (Fig.3).

A more serious mistake is an improper direction of hierarchical relations - Fig.6).

On base of this algorithm we grade the results of learners' work with 100-ball system. The experimental prototype of system that controls learners' knowledge by means of ontological analyses in URAN network was developed by Java.

\section{A MULTI-AGENT SYSTEM (MAS) FOR E-LEARNING: M(E)L (MULTI-AGENT E-LEARNING MAS)}

\section{A. A multi-agent system}

Parallel to the evolution of e-learning methodologies, the intelligent agent paradigm has generated such a remarkable interest in many application domains over the

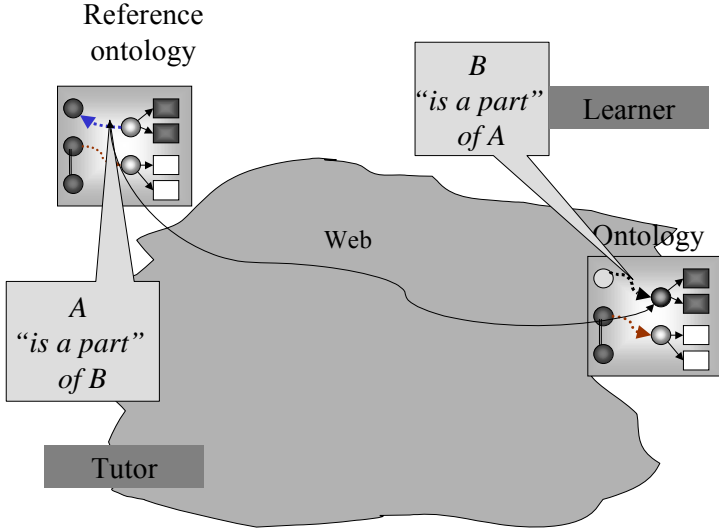

Figure 2. Hierarchical direction class error

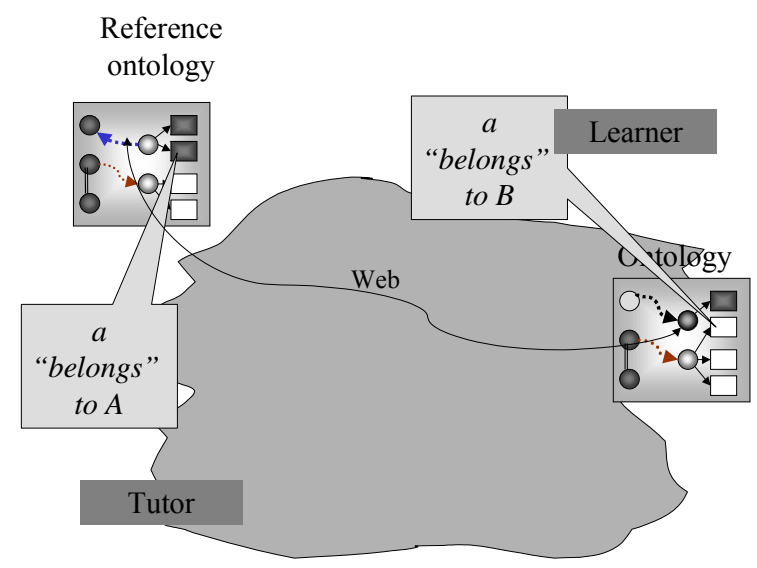

Figure 3. Instance classification error

last two decades. It is growing to be a continuously evolving and expanding area.

Ontological representation of learners' domain skills can be automatically processed by intelligent software agents [18]. It is appropriate to use software agents for elearning because they work efficiently in dynamic heterogeneous distributed environment [25]. One of the main properties of an intelligent agent is sociability. Agents are able to communicate between themselves, using some kind of agent communication language (for example, Knowledge Interchange Format - KIF or information transfer protocol Knowledge Query and Manipulation Language - KQML), in order to exchange any kind of information.

In that way they can engage in complex dialogues, in which they can negotiate, coordinate their actions and collaborate in the solution of a problem. However the model of languages for ontologies is based on specification XML, therefore FIPA has developed ACL for interaction of agents c support XML [26] that guarantees use the ontology language OWL for [27] for interaction with program agents.

A set of agents that communicate among themselves to solve problems by using cooperation, coordination and negotiation techniques compose a multi-agent system (MAS). A lot of researchers use MAS for e-learning and e-coaching tasks.

Various definitions from different disciplines have been proposed for the term multi-agent system (MAS). As seen 
from DAI (Distributed Artificial Intelligence), a multiagent system is a loosely coupled network of problemsolver entities that work together to find answers to problems that are beyond the individual capabilities or knowledge of each entity. More recently, the term multiagent system has been given a more general meaning, and it is now used for all types of systems composed of multiple autonomous components (agents) showing the following properties:

- Each agent has incomplete capabilities to solve a problem;

- There is no global system control;

- Data is decentralized;

- Computation is asynchronous;

One of the most important current factors promoting MAS development is the increasing popularity of the Internet, which provides the basis for an open environment where agents interact with each other to reach their individual or shared goals. To interact in such an environment, agents need to overcome two problems: they must be able to locate each other (since agents might appear, disappear, or move at any time); and they must be able to interact. Multi-agent systems are composed of two or more intelligent agents. An agent has detectors, effecters and a decision-making mechanism.

Using these mechanisms, a multi-agent-based simulation reproduces some phenomena inside online communities, including decision-making process of an agent, local interactions among agents and the system dynamics generated by local interactions, allowing us to observe and understand them [28]. One of the most interesting research topics is the organizational architecture styles of multi-agent system [29] where human organizations is used as a metaphor to suggest a set of generic styles for agent systems. Several architectural styles have been used in the development of multi-agent systems. [30] describes four such organizations:

- Hierarchical multi-agent systems

- Flat multi-agent systems

- Subsumption multi-agent system

- Modular multi-agent system

\section{B. A MAS for e-learning}

The dynamism in e-learning can be made more powerful with the help of intelligent agents. Intelligent agents -the so called e-assistants or helper programs - can reside inside a computer and make the learning in elearning occur dynamically to suit the need of the user. They can track the user's likes and dislikes in different areas, the level of knowledge and the learning style and accordingly recommend the best matching helpers for collaboration.

Personalized e-learning employs an active learning strategy which empowers the learner to be in control of the context, pace and scope of their learning experience. It supports the learner by providing tools and mechanisms through which he can personalize his learning experience $[31,32]$. This learner empowerment and shift in learning responsibility can help to improve learner satisfaction with the received learning experience.

The aims of personal e-learning agents are at increasing of information dissemination of existing courses through delivering the relevant course information offered to the right learner at the right moment. For example, learners' of different specialties learn on different programs and in many cases have different theoretical and practical background. Their personal agents can consider it and propose them not only the universal course program but additional facts and references from allied courses that they didn't learn.

Application of agent-based technologies in e-learning provides the personification of learners and trainers/ tutors and saved all users from the routine operations.

\section{An architecture for $M(e) L$}

An architecture of multiagent e-learning MAS M(e)L is proposed (Fig.4). It includes personal agents of learners and trainers. Use of some agents-facilitators raises the efficiency of this system and helps to users in search of required information. Agents of learners and trainers don't communicate directly. They send ontological information to informational agent that analyses them and returns the results to learner and trainer.

$\mathrm{M}(\mathrm{e}) \mathrm{L}$ prototype is a multiagent ontology-based elearning system that produces automatically semantic control of learners' domain beliefs of a course learnt. The focus of ontology analysis is on knowledge structuring (of main domain terms and their relations). Ontologies are used to describe learning materials and to represent learners' belief about course domain. M(e)L includes:

1) software agents of different types:

- personal learners' agents;

- personal trainers' agents;

- informational agent for communication support that facilitate users the DB and KB interaction;

2) knowledge base where ontological information about course is stored (repository of ontologies);

3) data base where personal information about users is stored;

Reference ontology is sent to $\mathrm{M}(\mathrm{e}) \mathrm{L}$ knowledge base by tutor personal agent.

LearnerAgent. Each learner has the corresponding Learner Agent that helps to the learning process of the learner. It acquires the learners' preferences and profile. During the learning process, as the learner enrolls in new courses, a dedicated learner agent for each course is created.

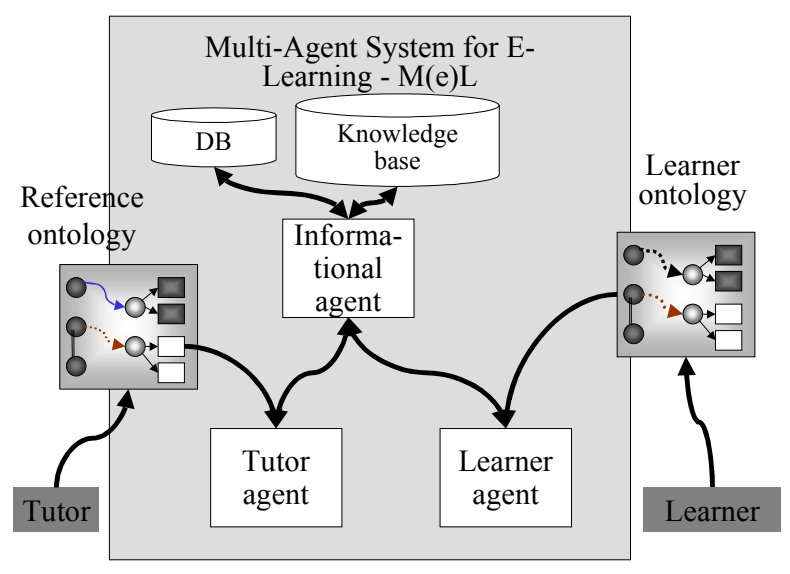

Figure 4. Architecture of e-learning MAS M(e)L. 
Trainer/Tutor Agent. The Trainer Agent assists the training process while interacting with the trainer. It is assigned for each trainer. For each course that is taught by the trainer a dedicated trainer agent is created. It provides training materials when requested by Informational Agent for distributing to learners' agents, assesses the progress and participation of different learners through quizzes, and manages the progress of the course. All the trainer agents of different courses of the same trainer are under the control of the Trainer Agent.

Informational Agent. The innovation in the proposed system is the introduction of the Informational Agent which is initialized as soon as any of the users starts to use the system. The Informational Agent plays a centric role in the proposed system. For each course, a dedicated Informational Course Agent is created. It has a collaboration mechanism which will be used for "matchmaking" and "community-building" to help increase collaboration between peers in a certain course. It also gives means to the trainer of the course to help in the training process such as statistics of the results of quizzes and summaries of learners' profiles to help in the final grading. It acts a mediator (facilitator) between Learner Agents and Trainer Agent of a specific course. After receiving the preferences (goals) of the trainer and the learners, it will run autonomously and self-dependently. All the Informational Agents of different courses are under the control of the Informational Manager Agent.

When a learner forms the domain ontology in OWL format her/his personal agent connects not with course trainer personal agent [33] but with informational agent and sends this ontology for comparing with reference one (its last version). After comparing the informational agent sends these results to the learners' and trainers' personal agent. If some learner or trainer usually prefers some way of learning and information presentation then the personal agent has to provide all these requirements for new course without direct instructions of the learner.

A learner receives information in an appropriate form and taking into account previous results of examination. For example, if a learner makes the same mistakes in ontology she/he receives the notification about it and advice that links with suitable course materials.

\section{Prototype implementation and future multi-agent technology}

In MAS E-Learning system three interconnected subsystems are allocated: learning, dialogue management (interaction) and ontology-based search of the learning resources and services.

In the subsystem of dialogue management it is necessary to create a system that returns the results of agent negotiations. The ontological subsystem provides intelligent search in distributed environment of the learning resources relevant for learning.

On the basis of the existing E-Learning MAS analysis [5-9] the requirements to software realization are formulated as follows:

1. Assurance of code portability on various platforms (UNIX, LINUX, Windows).
2. Availability of other platforms in a network. This requirement is a sequential of previous one. The mobile agents should carry out the work in heterogeneous computer environment.

3. Support of network interaction. Besides operations directly connected to moving between agent servers, the agent should have means for the communications with other agents and access to the removed resources.

Therefore support of network services should include a wide spectrum of opportunities (service of names, RPC, OLE, CORBA, RMI etc.).

4. Multiflow processing. For realization of synchronous execution of several actions MAS should include support of parallel agent function execution and support of synchronization means.

5. Safety. The mobile agents coming from the network can contain potentially dangerous, harmful code.

Therefore system should support safety means that are sufficient for its normal work. For development of logic model of MAS we use UML language. All intelligent agents of MAS are developed on a basis of Java class CIAgent which is in details described in [18]. The negotiations are begun by simple logic rules (in the terms if-then-else), and then pass to methods of rule formation that are based on the acquired facts.

In future using the inductive inference methods is planned to form the most appropriate personal strategy of learning for every learner (for example, some learners profit by theoretical materials and some other ones - from examples or practical tasks, somebody prefers graphical or text representation of information, etc.).

Use of learner personal agent allows to find the situation where a learner makes mistakes of the same type in ontologies of different courses. She/he receives the notification about it and advice that links with suitable logical course materials. Other important advantage of multiagent technology use is dealing with course tutor personal agent. If a big part of learners are making the same mistakes, a trainer receives the notification about it and can change suitable course materials.

\section{CASE: DOMAIN ONTOLOGY AND SEMANTIC WEB REPRESENTATION APPLIED IN AN E-LEARNING COURSE}

The challenges of e-learning MAS M(e)L were exploited in 2 e-learning courses in the university: in course "Visual C++ System Programming" (European University, Kiev, http://e-u.in.ua/eng/) and in the course "Modern Internet Technologies" (Kiev Slavistic University, http://www.mgi_ksu.edu.ua). Detailed domain models of these courses have been constructed. Model of course "Visual C++ System Programming" contains more then 150 terms and uses 8 relations between these terms. Course is accompanied by 16 online lectures and 10 practical exercises. Model of course "Modern Internet Technologies" contains more then 68 terms and uses 10 relations between these terms. Course is accompanied by 18 online lectures and 6 practical exercises (Table 1). 
PAPER

DOMAIn OnTOlogy, AN INSTRUMENT OF SEMANTIC WEB KNOWLEDGE MANAGEMENT IN E-LEARNING

TABLE I. AVERAGE MEASURES OF LEARNER ONTOLOGY PARAMETERS

\begin{tabular}{|l|c|c|}
\hline Course title & $\begin{array}{c}\text { "Visual C++ } \\
\text { System } \\
\text { Programmin } \\
\text { g" }\end{array}$ & $\begin{array}{c}\text { "Modern } \\
\text { Internet } \\
\text { Technologies } \\
"\end{array}$ \\
\hline Number of learners & 22 & 16 \\
\hline Number of terms & 153 & 68 \\
\hline Number of relations & 8 & 10 \\
\hline $\begin{array}{l}\text { Terms correctly used in learner } \\
\text { ontology K }\end{array}$ & $94.2 \%$ & $91.6 \%$ \\
\hline $\begin{array}{l}\text { Relations between terms correctly } \\
\text { used in learner ontology K }\end{array}$ & $72.0 \%$ & $66,3 \%$ \\
\hline $\begin{array}{l}\text { Type of relations between terms } \\
\text { correctly used in learner ontology } \mathrm{K}_{\text {type }}\end{array}$ & $89.1 \%$ & $81.5 \%$ \\
\hline mterm & 0.9 & 0.7 \\
\hline mrel & 0.3 & 0.5 \\
\hline miype & 0.7 & 0.8 \\
\hline $\begin{array}{l}\text { Overall rating of learner ontology } \\
\text { correctness K }\end{array}$ & $88,51 \%$ & $80,14 \%$ \\
\hline
\end{tabular}

$K=\left(K_{\text {term }} * m_{\text {term }}+K_{\text {rel }} * m_{\text {rel }}+K_{\text {type }} * m_{\text {type }}\right) /\left(m_{\text {term }}+m_{\text {rel }}+m_{\text {type }}\right)$

\section{A. Ontology of the domain of the course "The modern} internet technology for organizations".

$\mathrm{T}=\{$ Internet, network, local network, global network, metropolitan network, protocol, http, ip, tcp, ftp, address, service, Internet service, browser, hypertext, html, xml, agent, Web, Web service, UDDI, WSDL, SOAP, ontology, metadata, OWL, RDF, Semantic Web, ecommerce, e-learning, e-government, Protégé [21], RDFEdit ... $\}$.

$\mathrm{Rh}=\{$ “is a subclass", "is a part of", "includes" $\}$,

$\mathrm{Re}=\{$ “synonym", "another name of" $\}$,

$\mathrm{Rd}=\{$ "is used for", "defines by", "is a tool for", "is developed by", "is developed after"\}.

The fragment of this ontology made in Protégé is proposed in Fig. 5 .

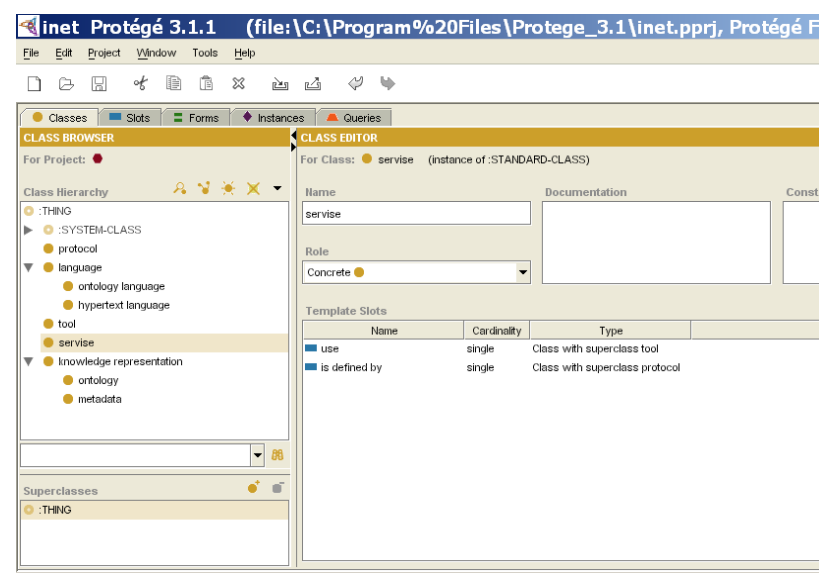

Figure 5. Representation of a course "The modern Internettechnologies" by means of the ontologies editor Protégé.

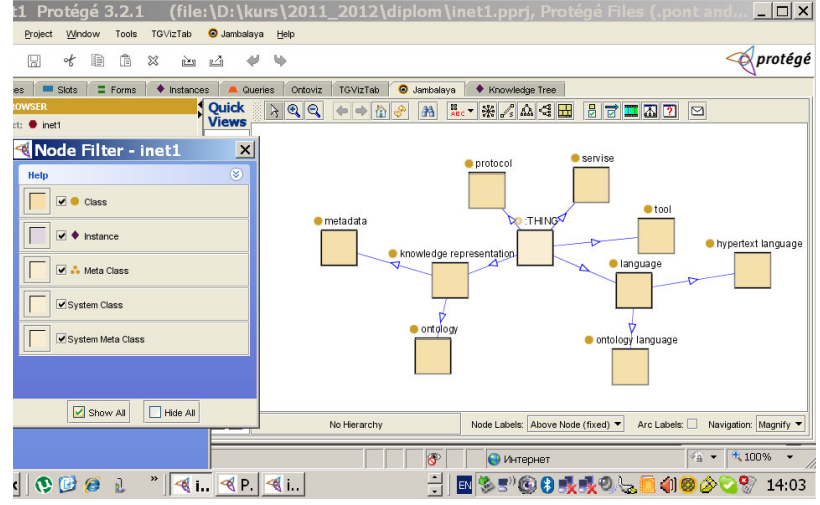

Figure 6. Ontology model of a course "The modern Internettechnologies" which is visualized with the help by means of the ontology editor Protégé.

This visual representation of reference ontology is generated by plug in Jambalaya of Protégé (Fig.6).

When learners receive the 4 sets of concepts and relations they have to build in Protégé the domain ontology and save it as an OW file. Then this ontology is matched with reference one.

B. $M(e) L$ in examination of learning and in support of the learner in the course "The modern Internettechnologies"

A first version of the multi-agent system has been implemented. Prototype of $\mathrm{M}(\mathrm{e}) \mathrm{L}$ was realized on base Java library of AWT-Abstract Window Toolkit (Fig.7).

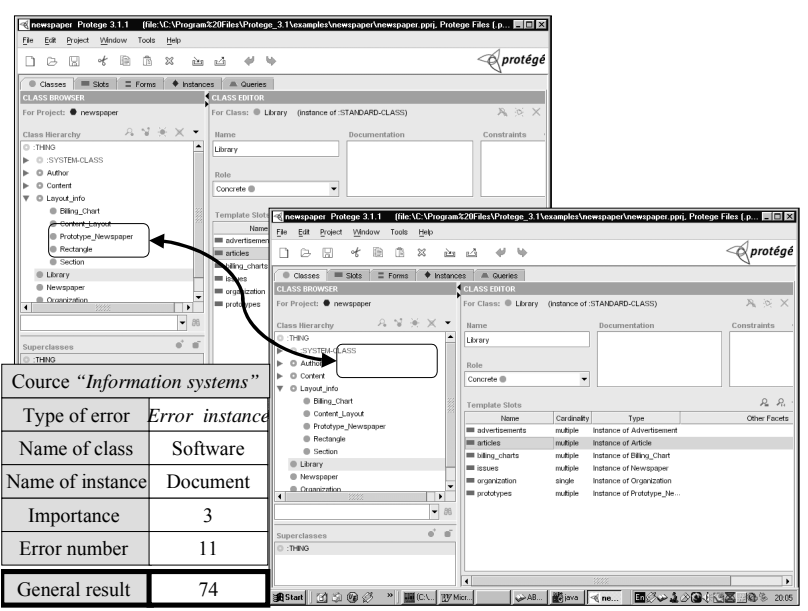

Figure 7. Domain ontology building and matching with reference one in $\mathrm{M}(\mathrm{e}) \mathrm{L}$

$K=\left(K_{\text {term }} * m_{\text {term }}+K_{\text {rel }} * m_{\text {rel }}+K_{\text {type }} * m_{\text {type }}\right) /\left(m_{\text {term }}+m_{\text {rel }}+m_{\text {type }}\right)$

\section{Discussion}

The agents in $\mathrm{M}(\mathrm{e}) \mathrm{L}$ are designed using a modified BDI (Beliefs, Desires and Intentions) architecture. Desires are determined first from the system requirements and then its intention and corresponding belief are found. This idea comes from the natural approach we usually do in the real world. Two kinds of use cases were used during designing of the system. External use cases are used for discovering the functions or services that the system should provide. Internal use cases are used for identifying plans 
(intentions), goals (desires), and their beliefs from the system services discovered from the external use case.

The main features of our approach to knowledge control in $\mathrm{M}(\mathrm{e}) \mathrm{L}$ are the following:

- all results are analyzed automatically without tutor;

- results are analyzed objectively;

- learners can work with knowledge base;

- a structurization of domain knowledge simplifies the learning process;

- tutors can exchange their knowledge based on reference ontologies.

One of the essential elements needed for effective learning is feedback. In the current generation of elearning systems automatically produced feedback is almost only used in question-answer situation. Valuable feedback, for example produced by a human tutor via email, is often possible but this introduces delays and is time consuming. We want to develop ontology-based mechanisms of feedback that use the context of education. Different learner errors need different methodologies of tutor to describe their causes.

Feedback is used in many learning paradigms. The concept of feedback is very important in educational psychology. It is one of the main psychological principles that one of the essential elements needed for effective learning is feedback. Information about examining results is required to assess progress, correct errors and improve performance. Feedback describes any communication or procedure given to inform a learner of the accuracy of a response, usually to an instructional question. Feedback allows the comparison of actual performance with some set standard of performance. Information that is acquised by learner from feedback instruction includes not only answer correction but other information such as precision, timeliness, learning guidance, motivational messages, background material, sequence advisement, critical comparisons, and learning focus. In traditional learning learners and tutors can interact directly and learners can freely ask questions and tutors usually know whether their learners understand concepts or problem solving techniques and relations between them. Feedback is an important component of this interaction. In e-learning systems feedback problem is much more difficult and has a lot of technological and social aspects.

\section{CONCLUSION}

Intelligent control of learners' skills and ability for retrieval of new information relevant to subject domain of learning are the important elements of effective learning. We propose to use the domain ontology as an instrument for automated examining of learners' skills on semantic level. Learners have to build a personal domain ontology and thesauri of the knowledge domain (on base of natural language texts relevant to subject domain -books, journals, manuals etc.) and then compare them with reference ones that are built by a trainer/ tutor. Analysis of learners' mistakes allows to propose them personalised recommendations and to improve the course materials in general. A personal ontology will be improved in the process of learning and can expand the reference ontology according to personal skills and knowledge of the learner (for example, by terms in other languages known to the learner). In future this ontology can be used for personalized semantic informational retrieval in corresponding subject domain for defining of a learners' sphere of interests and in professional activity - for describing the learners' competences.

A formal model of ontology for e-learning is set forward and the method of developing ontology and the principles of ontological analysis are explained. Evaluation of the learners' knowledge results from comparing the learners' ontology with the reference ontology of the trainer/ tutor. A multi-agent system for elearning $(\mathrm{M}(\mathrm{e}) \mathrm{L})$ has been defined and a prototype system has been developed. In a case, the domain ontology and the semantic web representation has been applied in 2 elearning courses in the university.

In future, we plan to develop a more powerful algorithm of ontology analyses that will consider ontology integration and its distributed upgrade on base of multiagent technologies. Application of learners and tutor agents will provide the personalization of distributed learning process. These agents will use the history of learning for feedback between learner and trainer/ tutor.

\section{ACKNOWLEDGEMENTS}

This work was supported in part by the Grant NATO NIG 971779. "National Telecommunication Networks for Scientific and Educational Institutions of Ukraine with Access to Internet - URAN" [34] and EU INCOCopernicus Project 960114 - EXPERNET "A distributed Expert System for the Management of a National Network of Ukraine".

This work was supported in part by Kelly Lada de Mandraka that don't disturb the authors along the research work and article editing.

\section{REFERENCES}

[1] McArthur, D., Lewis, M., and Bishay, M. The Roles of Artificial Intelligence in Education: Current Progress and Future Prospects. 1993. - http://www.rand.org/hot/mcarthur/ Papers/role.html.

[2] Forbus, K. D. and Feltovich, P. J. The Coming Revolution in Educational Technology. Smart Machines in Education, eds. Forbus, K. D. and Feltovich, P. J., pages 3-5, AAAI Press/MIT Press, 2001.

[3] Aroyo, L., Dicheva, D., Courseware authoring tasks ontology // Proceedings of the international conference in education, IEEE, 2002.

[4] T. Murray, S. Blessing, S. Ainsworth, Authoring tools for advanced technology learning environments: towards costeffective adaptive, interactive, and intelligent educational software. - http://helios.hampshire.edu/ tjmCCS/atoolsbook/chap tersV2/ChapterList.html.

[5] Kay, J. Learner Control. User Modeling and User-Adapted Interaction, 11:111-127, 2001. http://dx.doi.org/10.1023/A:101119 $\underline{4803800}$

[6] IEEE Learning Technology Standards Committee (LTSC): http://ltsc.ieee.org/

[7] Magnin L., Snoussi H., Nie J., Toward an Ontology-based Web Extraction, The Fifteenth Canadian Conference on Artificial Intelligence, 2002.

[8] Bredeweg B., Forbus K. D. Qualitative Modeling in Education. AI Magazine, V. 24, No. 4.

[9] Angelova A., Kalaydjiev G., Strupchanska A. Domain Ontology as a Resource Providing Adaptivity in e-learning. Iml.bas.bg/ albena/publications/wose_paper5.pdf.

[10] Berners-Lee, T., Hendler, J., \& Lassila, O. The Semantic Web. Scientific American 284, 34-43, May 2001. http://dx.doi.org/10.1038/scientificamerican0501-34

[11] Decker, S., Melnik, S., van Harmelen, F., Fensel, D., Klein, M., Broekstra, J., Erdmann, M., \& Horrocks, I. The Semantic Web: 
The Roles of XML and RDF. IEEE Internet Computing 4, 63-74, Sep./Oct. 2000. http://dx.doi.org/10.1109/4236.877487

[12] Wooldrige M., Jennings N.R. Intelligent Agents: Theory and Practice / Knowledge Engineering Review, Vol.10, No.2, 1995.P.115-152. http://dx.doi.org/10.1017/S0269888900008122

[13] Gómez-Pérez, A., \& Corcho, O. Ontology Languages for the Semantic Web. IEEE Intelligent Systems 17(1), 54-60, 2002. http://dx.doi.org/10.1109/5254.988453

[14] Devedzic, V. Knowledge Modeling - State of the Art. Integrated Computer-Aided Engineering 8, 257-281, 2001.

[15] Heflin, J., \& Hendler, J. A Portrait of The Semantic Web in Action. IEEE Intelligent Systems 16(2), 54-59, 2001. http://dx.doi.org/10.1109/5254.920600

[16] Resource Description Framework (RDF) Model and Syntax Specification, W3C Recommendation. - http://www.w3.org/TR/ REC-rdf-syntax/.

[17] OWL. - http://www.w3c.org/TR/owl-features/.

[18] Gladun A., Rogushina J., Shtonda V. Ontological Approach to Domain Knowledge Representation for Informational Retrieval in Multiagent Systems // International Jornal "Information Theories and Applications", V.13, N.4, 2006. - P.354-362.

[19] Gladun A., Rogushina J., An Ontology-Based Approach to Student Skills in Multiagent E-learning Systems // Information Technologies \& Knowledge, vol.1, №3, 2007.-P.219-225.

[20] Swartout, W., \& Tate, A. Ontologies, Guest Editors' Introduction. IEEE Intelligent Systems 14(1), 18-19, 1999. http://dx.doi.org/10.1109/MIS.1999.747901

[21] Protégé - http://protege.stanford.edu/ontologies/ontologyOfScienc $\underline{\mathrm{e}}$

[22] IDEF5 Overview. - http://www.idef.com/IDEF5.html.

[23] T. Murray S. Blessing, S. Ainsworth, Authoring tools for advanced technology learning environments: towards costeffective adaptive, interactive, and intelligent educational software. - http://helios.hampshire.edu/ tjmCCS/atoolsbook/ chaptersV2/ChapterList.html.

[24] Gladun A., Rogushina J. Multiagent ontology-based intelligent system of e-commerce // The International Conf. TAAPSD'2004. Bulletin of Taras Shevchenko University. A series "Physicomathematical sciences ", 2004. - C.118-124.

[25] Gritsenko V., Gladun A., Rogushina J. Agent-oriented Programming as a Tool of the Intellectual Distributed Applications Development. Proc. of International Scientific Conference "Software Design: Challenge of Time and Role in Informational Society", 2005.

[26] The FIPA Standard for Interoperating Software Agents. The Foundation for Intelligent Physical Agents, 2000, www.fipa.org

[27] Holger Knublauch, Mark A. Musen, Natasha F. Noy Creating Semantic Web (OWL) Ontologies with Protege // Tutorial: 2nd
International Semantic Web Conference (ISWC2003), Sanibel Island, Florida, USA, October 20-23th, 2003

[28] Gladun A., Rogushina J. Knowledge Management in the Clinical Multiagent Information System. Proc. of International Conf. Advanced Information and Telemedicine Technologies for Health AITTH 2005, V.2., Minsk. - 2005. - P. 76-80.

[29] Gladun A., Rogushina J. Ontologies as a Perspective Direction of Intellectualization of Informational Retrieval in Multiagent Systems of E-commerce // The Proceedings of XI-th Intern. Conf. "Knowledge-Dialogue-Solution", KDS'2005, Varna, Bulgaria, pp.112-120.

[30] FIPA Abstract Architecture Specification, 2002. http://www.fipa.org/specs/fipa00001/index.html

[31] Gladun A., Rogushina J. Use of Semantic Web Technologies and Multilinguistic Thesauri for Knowledge-Based Access to Biomedical Resources // International Journal of Intelligent Systems and Applications, 2012, №1, P.11-20.

[32] Gladun A., Rogushina J. Mereological Aspects of Ontological Aalysis for Thesauri Constructing // In Book "Building and Environment", v.5, 2008, Nova Publishing, New-York, USA.P.89-103.

[33] Gladun A., Rogushina J. Use of Ontological Analysis for Student Skills Control in E-Learning: Semantic Web Approach // International Journal of Engineering and Technology, V.1 No. 3, December, 2011.

[34] Ukrainian Research and Academic Network (UARDNET). http://www.cei.uran.net.ua/eng/uran.htm.

\section{AUTHORS}

A.J. Gladun works in International Research and Training Centre of Information Technologies and Systems, National Academy of Sciences and Ministry of Education of Ukraine, 44 Glushkov Pr., Kiev, 03680, Ukraine; (e-mail: glanat@yahoo.com).

J.V.Rogushina works in Institute of Software Systems, National Academy of Sciences of Ukraine, 44 Glushkov Pr., Kiev, 03680, Ukraine; (e-mail: ladamandraka2010@gmail.com).

J. Schreurs is emeritus professor of Hasselt University. Hasselt University, Agoralaan, 3590 Diepenbeek, Belgium. www.uhasselt.be, jeanne.schreurs@uhasselt.be, mob. +32475497099 .

Received 26 September 2012. Published as resubmitted by the authors 14 November 2012. 